\title{
The Pattern of Ocular Morbidity, Findings from a Study Conducted in Western Remote Hilly Region of Nepal
}

\author{
Bastola $\boldsymbol{P}^{*}$

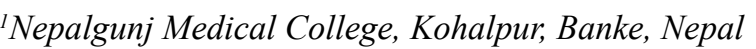

\section{* Corresponding Author: \\ Dr. Pradeep Bastola \\ Consultant Ophthalmologist, Assistant Professor Nepalgunj Medical College, Kohalpur, Banke, Nepal. Email: pbs_dinku@yahoo.com}

\section{Citation}

Bastola P.The pattern of Occular morbidity, findings from a study conducted in western remote hilly region of Nepal. Nepal Journal of Medical Sciences. 2012; 1(1): 35-38.

\section{Background:}

$\mathrm{V}_{\mathrm{i}}$ significant socioeconomic impact. Childhood blindness is a priority area because of the number of years of blindness that ensues. Data on the prevalence and causes of blindness and severe visual impairment in children are needed for planning and evaluating preventive and curative services for children, including planning special education and low vision services. The available data suggest that there may

\begin{abstract}
Background: There is a lack of study in the pattern of ocular morbidity in western hilly region of Nepal. Therefore a study was carried out to find out the pattern of ocular morbidity, in various age groups in a diagnostic, screening and treatment camp (DST) held in a western hilly remote region of Nepal.
\end{abstract}

Methods: A descriptive, cross sectional study was done to find out the pattern of ocular morbidity in a western hilly region of Nepal. All the cases included in the study were examined thoroughly from Ophthalmological perspective and a diagnosis was made. Patients needing treatment both medical as well as surgical were advised accordingly and referred when indicated.

Results: A total of 712 eyes of 356 cases were examined in this study. The range of the age varied from two months of life to 91 yrs of age. Age wise most number of cases were in the school going age group accounting for almost $80 \%$ of all the cases. The commonest ocular morbidity encountered were refractive error $(15.4 \%)$, dry eyes, ocular allergy, followed by cataract. Majority of the cases had both dry eyes and ocular allergy. While 70 eyes of 35 patients had no ocular morbidity.

Conclusion: The lower prevalence of age related cataract was due to the extensive cataract surgical coverage provided in the region by various organizations and because of lesser number of cases examined above the age of 40 years. Finding of just three cases of Xerophthalmia (X2B), pointed out towards the success of extensive Vitamin A supplement program run by the ministry of health.

Keywords: Refractive error; dry eyes; ocular allergy; cataract 
surveys depicting the magnitude of ocular diseases among the elderly population in Nepal are few. ${ }^{2}$

Any information on eye diseases in Nepal is rare and sketchy. A program needs to provide basic eye screening to school children as well as patients in other age groups with an aim to provide services as well as gather information on ocular morbidity. ${ }^{3}$

This study aimed at finding out the current pattern of ocular morbidity in a rural hilly community of Nepal in western region and to prevent the preventable causes of blindness and to cure the curable causes of blindness, so as to achieve the World Health Organization goal of achieving vision $20 / 20$ by the year 2020 .

\section{Methods:}

This was a cross sectional, descriptive type of study, carried out by Gandaki Medical College and Research Centre, Charak Hospital, Pokhara, Nepal, in a diagnostic screening and treatment (DST) camp held in a rural western hilly region of Baglung district, Nepal; with around 80,000 population to be covered, in the year 2010. The study was approved by the ethical committee. All the patients attending the eye out patient department (OPD) were included in the study including the referred cases from other out patient departments too. An informed consent was taken from the patients.

In all cases a complete thorough ocular examination was carried out starting from visual acuity to complete evaluation of the fundus after dilatation. In cases needing special investigations, they were carried out like Schirmer's test, Tear film break up time, fluroscein staining of the cornea, intraocular pressure measurement, evaluation of ptosis, evaluation of proptosis, syringing and probing, to find out and to rule out the patency or blockage of the lacrimal drainage system. Similarly retinal function tests and orthoptic tests were carried out in needy cases. Cases needing intervention like cases of chalazia, drainage of external hordeolum, and epilation of the eye lashes in cases of trichiasis were treated in the minor operation theatre in the examination spot itself. While cases needing further investigations and surgical intervention were referred to Gandaki Medical College, Pokhara like cases of cataracts, pterygium, ptosis, strabismus etc. Medical treatment for the cases, glasses prescription was provided in the screening camp itself. Cases needing referral to other departments were referred accordingly. All the cases were entered in a profroma specifically designed for the study. Statistical analysis was done using statistical package for social service (SPSS) version 11.5. A statician was consulted when necessary.

\section{Results:}

A total of 712 eyes of 356 patients were examined. Gender wise 207 patients were female which accounted for $58 \%$ of the total patients, similarly $149,(42 \%)$ of the patients were male patients. Age wise most number of cases were in the school going age group accounting for almost $80 \%$ of all the cases examined (Table 1). While 55 cases examined that is $15.4 \%$, were in the age group beyond 40 years of age.

Table 1: Age wise distribution of the patients

\begin{tabular}{lcc}
\hline Age group & Number & Percent (\%) \\
\hline $0-9$ & 11 & 3.1 \\
$10-19$ & 266 & 74.7 \\
$20-29$ & 13 & 3.7 \\
$30-39$ & 11 & 3.1 \\
$40-49$ & 19 & 5.3 \\
$50-59$ & 10 & 2.8 \\
$60-69$ & 16 & 4.4 \\
$70-79$ & 9 & 2.5 \\
$80+$ & 1 & 0.3 \\
Total & 356 & 100 \\
\hline
\end{tabular}

The commonest ocular morbidity encountered after ocular examination was refractive error, followed by dry eyes, ocular allergy, cataract, degenerative conjunctival conditions, corneal opacity etc.

In 70 eyes of 35 cases no ocular abnormality was detected. Surprisingly, only 3 case of Vitamin A deficiency were diagnosed, all the cases were of Xerophthalmia X2B.

Table 2: Pattern of ocular morbidity

\begin{tabular}{lll}
\hline Types of Ocular morbidity & $\begin{array}{c}\text { Number } \\
\text { of cases }\end{array}$ & Percent (\%) \\
\hline Refractive error & 55 & 15.4 \\
Dry eyes & 50 & 14 \\
Ocular allergy & 40 & 11.2 \\
Age related cataract & 21 & 5.9 \\
Corneal opacity & 18 & 5.1 \\
Pterygium & 15 & 4.2 \\
Sqaumous blepharitis & 15 & 4.2 \\
Pinguencula & 13 & 3.7 \\
Chronic dacryocystitis & 10 & 2.8 \\
Conjunctival naevus & 10 & 2.8
\end{tabular}




\begin{tabular}{lll} 
Conjunctivitis & 8 & 2.2 \\
Asthenopia & 7 & 1.9 \\
Chalazion & 7 & 1.9 \\
Diabetic retinopathy & 7 & 1.9 \\
Solar maculopathy & 7 & 1.9 \\
Strabismus & 5 & 1.4 \\
Hordeolum externum & 5 & 1.4 \\
Psuedophakia & 4 & 1.1 \\
Hypertensive retinopathy & 3 & 0.8 \\
Xerophthalmia & 3 & 0.8 \\
Congenital ptosis & 3 & 0.8 \\
Herpes zoster Ophthalmicus & 3 & 0.8 \\
Computer vision syndrome & 2 & 0.6 \\
\hline
\end{tabular}

\section{Discussion:}

In the study, according to the results; around $80 \%$ of the patients were of school going age and coincidentally, the commonest ocular morbidity encountered was refractive error, which accounted for $15.4 \%$ of the all ocular morbidity encountered. The Nepal Blindness Survey (NBS) found refractive error, based on pinhole correction to be $1.3 \%{ }^{4}$ Another study conducted in eastern Nepal found refractive error in school children to be less than 3\%.5 This study differed from Nepal blindness survey as well as the other study conducted in eastern Nepal because refractive error in the above mentioned studies accounted for minor proportion of ocular morbidity. Our study has shown increased number of cases of refractive error, this shows the changing pattern of ocular morbidity in Nepal from those days to the present era. It may also be because of higher number of school going children in our study.

The Nepal Xerophthalmia Survey, also conducted in 1981, showed that $1.7 \%$ of children below 14 years of age had Bitot's spot (X2B) presumed to be due to vitamin A deficiency. ${ }^{6}$ While in this study, $0.9 \%$ of the total examined patient showed ocular signs of vitamin A deficiency. The reduction in the prevalence of Xerophthalmia is probably due to national vitamin A supplement program run by the ministry of health.

A very interesting as well as surprising finding of the study was not to find a single case of trachoma or its sequalae, which may be due to the various programs run by Nepal Netra Jyoti Sangh (NNJS) and Ministry of Health for trachoma throughout Nepal. Surgery, Antibiotics, Face wash and Environmental sanitation (SAFE) strategy to prevent the occurrence and treat trachoma cases, which is effective in the region where the study was conducted. While similar study done in India in school going children in a rural community showed to have, trachoma as the most common cause of ocular morbidity, which accounted for $18 \%$ of the total study population.?

The prevalence of dry eyes, ocular allergy, conjunctival degenerations and corneal opacity was very high in the study, which may be due to the age group examined and the agricultural background of the patients mostly having sun exposure in the field as well as work in the field mostly. The prevalence of cataract in the study was found to be very low as compared to other studies, like Nepal blindness survey, where cataract was the commonest ocular morbidity as well as the commonest cause of blindness in Nepal. ${ }^{4}$ While the recent blindness surveys done in Nepal, like Gandaki blindness survey, Bheri blindness survey as well as Lumbini blindness survey they all have found cataract still the leading cause of ocular morbidity as well as the commonest cause of blindness in Nepal. ${ }^{8}$ This low prevalence rate of cataract in the study group was probably due to younger age group of study population. A part from that present study has provided the common pattern of ocular morbidity in a rural hilly region of Nepal.

We hope this kind of study would help in planning and making strategy for implementing certain eye programs in rural hilly regions of Nepal. This kind of study would always be helpful to find out the changing pattern of ocular morbidity in Nepal in different parts. Most heartening finding of the study was, to see that we are in the right path to achieve the goal of "the right to sight" the moto of vision $20 / 20$ by 2020 , as the study showed the prevalence of preventable, treatable as well as curable ocular diseases were low.

\section{Conclusion:}

Refractive error along with dry eyes and ocular allergy topped the list because majority of the cases were in the age group of 10 to 19 years. The lower prevalence of age related cataract was may be due to the extensive cataract surgical coverage provided in the region by various organizations and mainly because of lesser number of cases examined above the age of 40 years. Finding of just three cases of Xerophthalmia (X2B), pointed towards the success of extensive Vitamin A supplement program run by the ministry of health in Nepal. This kind of cross sectional study helps to find out the magnitude of eye problem in various age groups and is advised to be conducted in different regions of Nepal as well as Indian sub continental region, and will be helpful to find out the changing pattern of ocular morbidity and would help to achieve the goal of vision $20 / 20$ by 2020 . 
Original Article | Bastola P. Occular morbidity in remote Western Nepal.

\section{Acknowledgement:}

We would like to acknowledge all the patients and their care takers for helping us out in completing this study similarly; we would like to acknowledge the entire team of Gandaki Medical College who directly or indirectly took part in this diagnostic, screening and treatment camp held by Charak Hospital, Pokhara Nepal and at the mean time we would like to that Professor D.S. Sharma and his team for approving this study from the ethical approval committee and helping me out to carry out this study.

\section{References:}

1. Gupta M, Gupta PB, Chauhan A, et al. Ocular morbidity prevalence among school children in Shimla, Himachal, North India. Community Eye Care 2009;57:133-8.

2. Atraman R, Rao SP, Nayar SO, et al. Ocular morbidity among elderly population in a rural area of central India. Community Eye Care 2009;58:133-8.

3. Nepal BP, Koirala S, Adhikari S, et al. Ocular morbidity in school children in Kathmandu. Br J Ophthalmol 2003;87:531-4.
4. Brilliant GE. Xerophthalmia, Keratomalacia and Childhood blindness. The epidemiology of blindness in Nepal: Report of the 1981 Nepal Blindness Survey. Chelsea, Michigan: the Seva Foundation 1988;8:1-69.

5. Pokharel GP, Negrel AD, Munoz SR, et al. Refractive error study in children: results from Mechi Zone, Nepal. Am J Ophthalmol 2000;129:436-44.

6. Upadhyay MP, Gurung BP, Pillai KK. Xerophthalmia among Nepalese children. Am J Epidemiol $1985 ; 121: 71-7$.

7. Chaturvedi S, Agrawal OP. Pattern and distribution of ocular morbidity in primary school children of rural Delhi. Asia Pac J Public Health 1999;11:30-3.

8. Sherchan A, Kandel RP, Sharma MK, et al. Blindness prevalence and cataract surgical coverage in Lumbini Zone and Chitwan District of Nepal. Br J Ophthalmol 2010;94:161-6. 